\title{
Implementation of Pilot Channel Estimation Techniques for OFDM System with Low Mean Square Error
}

\author{
T. Padmavathi ${ }^{1}$, Dr. Kusma Kumari Cheepurupalli ${ }^{2}$ and Dr. R. Madhu ${ }^{3}$ \\ ${ }^{1}$ Sr.Asst. Professor, CVR College of Engineering /ECE Department/Hyderabad, India \\ Email: padmatp41@gmail.com \\ ${ }^{2}$ Assoc. Professor, GVP College of Engineering, (Autonomous), /ECE Department, Visakhapatnam, India \\ Email: chkusumasrinivas@gmail.com \\ ${ }^{3}$ Asst. Professor, University College of Engineering, JNTUK/ECE Department, Kakinada, India \\ Email: madhu ramarkula@rdiffmail.com
}

\begin{abstract}
Orthogonal Frequency Division Multiplexing (OFDM) has grown to be the most popular multi carrier communication system in high speed data communications. The available bandwidth is utilized efficiently, and more data rates can be achieved because of orthogonal carriers in OFDM system. OFDM system requires precise channel estimation and synchronization to reduce Mean Square Error (MSE). In this work, various Pilot Channel Estimation (PCE) techniques are used to compute CSI (Channel State Information). Pilots are transmitted along with the data and channel response is computed with various interpolation techniques such as linear, spline, cubic Interpolation techniques by extracting the pilots at the receiver. The performance metrics like Mean Square Error (MSE) and computation time are computed and compared for different PCE techniques. Discrete Fourier Transform (DFT) based channel Estimation is combined with interpolation techniques and channel response is computed and compared with Least Squares (LS) and Minimum Mean Square Error (MMSE) approaches. LS, MMSE, LMMSE (Linear MMSE) methods are combined with Time Division(TD) and Time Division Duplexing(TDD) techniques to compute MSE. TD-LMMSE approach achieves least MSE with more computation time.
\end{abstract}

Index Terms: OFDM, channel estimation, interpolation, CSI, MSE, computation time.

\section{INTRODUCTION}

OFDM (Orthogonal Frequency Division Multiplexing) divides the entire channel bandwidth into subchannels, which increases the symbol duration. OFDM eliminates the Inter Symbol Interference (ISI) caused by multipath environment due to increased symbol duration [1]-[3]. Wireless LAN such as IEEE802.11a and HIPERLAN/2 and multimedia communications are implemented with OFDM.

Multipath propagation introduces constructive and destructive Interference at the receiver. Since the wireless channel is time varying and frequency selective, a dynamic Channel estimation is necessary before demodulation of OFDM signal [4]. Channel estimation can be done with two approaches. Blind channel estimation requires less bandwidth but it depends on only the statistical information of received symbols [5]. Pilot Channel Estimation inserts the pilots in the data sequence and consumes more bandwidth. In OFDM, either entire OFDM symbol is used for pilot transmission or pilot symbols may be inserted periodically after group of OFDM symbols [6]. The first method is, block type pilot channel estimation and it has been developed for slow fading channel. Least Squares (LS) and Minimum Mean Square Approaches (MMSE) has been used for Block-Type pilot arrangement to compute Mean Square Error (MSE). Performance of MMSE Method is better in terms of MSE than the LS but is more complex. [7]. Combtype pilot channel estimation has been introduced to equalize the fast fading channel. The algorithms have been implemented for Comb-Type channel estimation is based on interpolation since the pilots are characterized in the frequency domain. In comb-Type channel estimation, channel can be estimated at the pilot frequencies based on LS, MMSE. The performance of MMSE is better than LS[8,9], and the complexity of MMSE is reduced by singular Value Decomposition low rank estimator. Low rank channel estimators proposed for comb-type pilot arrangement reduces computational time but it requires memory [10]. In the spectrum of OFDM system, the subcarriers of symbol carries data and pilots, these pilot carries are interpolated in time domain to estimate the channel. But time domain interpolation fails to keep track of quick channel variation caused by fast moving receiver. Even though channel estimation without time-axis interpolation enables fast estimation, the performance of channel estimation degraded for a long delay spread channel in a single carrier networks (SCNs) because of inadequate pilot sub-carriers [11]

Channel estimation can be done with the help of Wavelets to reduce the MSE. It improves Transmission efficiency and Date rate because channel coefficients will be estimated in time domain effectively. But the accuracy of proposed Haar wavelet based channel estimation need to be improved [13].

In frequency domain, OFDM signal can be represented with group of subcarriers. Each subcarrier frequency response can be obtained by interpolation techniques. In comb-type channel estimation, Channel can be interpolated with linear interpolation, spline Interpolation, Cubic interpolation and time domain interpolation. Performance of the interpolation techniques are compared in terms of MSE by including different modulation techniques like BPSK, QPSK. OFDM System model and its mathematical representation are described in Section II. In section III pilot channel estimation with various interpolation techniques are discussed. Simulation Results are discussed in Section IV. 


\section{System ModeL}

$$
Y(k)=F F T\{y(n)\} \text { for } k=0,1,2 \ldots \ldots . . N-1
$$

The pilot channel estimation based OFDM system given in Figure.1. The binary data is mapped to constellation points using BPSK/QPSK modulations. Pilots are inserted

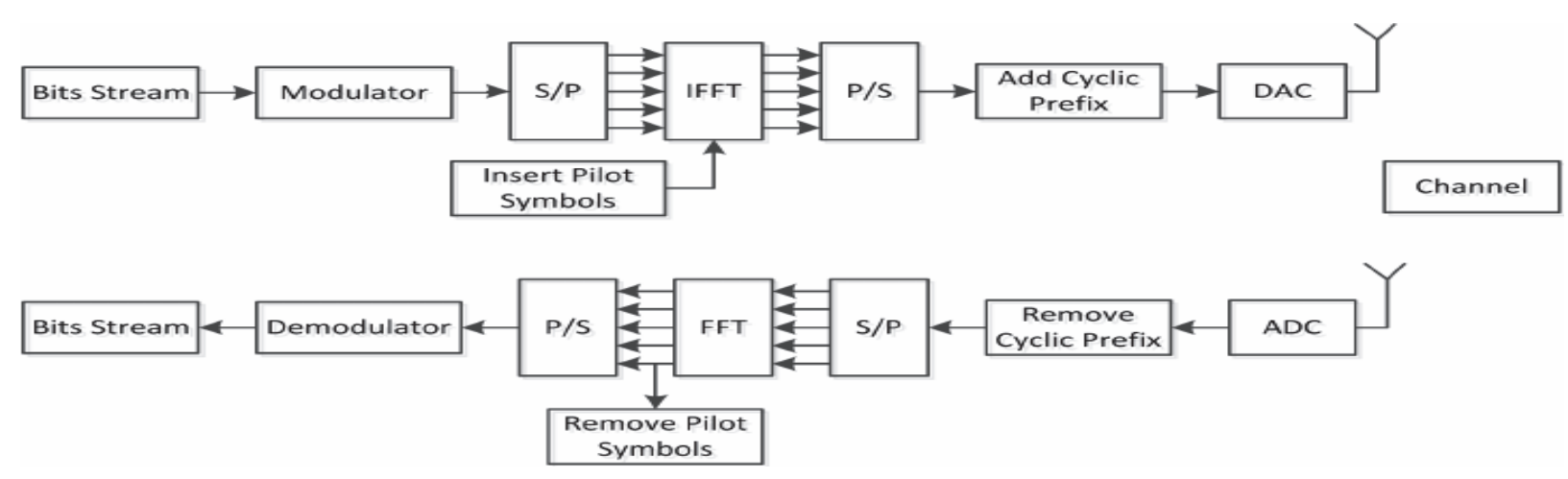

Figure.1 OFDM System

either to all OFDM Symbols periodically or only for specific symbols. The resulting data converted from frequency domain to time domain using IFFT with the following equation

$$
\begin{aligned}
x(n) & =\operatorname{IFFT}(X(K)) \text { for } n=0,1, \ldots \ldots \ldots . N \\
& =\sum_{K=0}^{N-1} X(K) e^{\left(\frac{j 2 \pi k n}{N}\right)}
\end{aligned}
$$

Where $\mathrm{N}$ is the FFT length. Guard time is added to $\mathrm{x}(\mathrm{n})$ which is to be larger than the expected delay spread, so that Inter-Symbol Interference is removed. Part of OFDM symbol is cyclically extended with the guard time, to remove Inter Carrier Interference (ICI). Then resulting signal is given by

$$
x_{f}(n)= \begin{cases}x(N+n), n=-N_{g}, N_{g}+1, \ldots \ldots \ldots, 1 \\ x(n) & n=0,1,2 \ldots \ldots \ldots N-1\end{cases}
$$

Where $\mathrm{Ng}$ is the guard time interval. The signal form transmitter is passed through the frequency selective fading channel with Additive White Gaussian Noise (AWGN). In this paper frequency selective fading channel is modelled with Rayleigh fading. The signal at the receiver is given by $y(n)=x_{f}(n) \otimes h(n)+w(n)$

Where $\mathrm{y}(\mathrm{n})$ is the received signal, $\mathrm{h}(\mathrm{n})$ is the Impulse response of the channel, w(n) is the AWGN Noise. Channel Impulse Response (CIR) h is given by

$$
h(n)=\sum_{r=0}^{m-1} h_{r} e^{j\left(\frac{2 \pi}{N}\right) f_{D r} T_{n} \delta\left(\lambda-\tau_{r}\right)} \quad \text { for } 0 \leq n \leq N-1
$$

where $\mathrm{m}$ is the total propagation paths, $\mathrm{h}_{\mathrm{r}}$ is the complex impulse response of the $\mathrm{r}^{\text {th }}$ path, $f_{\text {Dr }}$ is the Doppler frequency shift, $\lambda$ is the delay spread index, $\mathrm{T}$ is the sample period and $\tau_{\mathrm{r}}$ is the $\mathrm{r}^{\text {th }}$ path delay. The signal received at the receiver after passing through the A/D converter and low pass filter, guard time is eliminated then

$$
y(n)=y_{f}(n) \text { for }-N_{g} \leq n \leq N-1
$$

$\mathrm{Y}(\mathrm{k})$ is obtained by sending $\mathrm{y}(\mathrm{n})$ to the FFT block,

$$
1 / N \sum_{n=0}^{N-1} y(n) e^{-j\left(\frac{2 \pi k n}{N}\right)}
$$

Assume there is no ISI [8], then $\mathrm{H}(\mathrm{k})$ can be obtained from the relation

$$
H(k)=\operatorname{IFFT}(h(n))
$$

After IFFT block, in the channel estimation block pilots are extracted and estimated for data sub-channels. Then the estimate of data is given as

$X_{\theta}=\frac{Y(k)}{H_{\theta}(k)} \quad k=0,1, \ldots . . N-1$

\section{IMPlementation OF PILOT ChanNel ESTIMATION TECHNIQUES}

Pilots symbols are transmitted periodically and all subcarriers carries pilot symbols in PCE. The channel is assumed to be constant during the pilot transmission, resulting no error in the channel estimation. PCE can be performed by either LS or MMSE approach [7], [9]

The signal at the receiver can be given by the following equation in the absence of ISI

$$
Y(k)=X(k) H(K)+W(n)
$$

In the matrix form above equation can be represented as $Y=X F h+W$

Where

$$
\begin{aligned}
& X=\operatorname{diag}(X(0), X(1), \ldots \ldots, X(N-1) \\
& Y=[Y(0), Y(1), \ldots \ldots \mathrm{Y}(\mathrm{N}-1)]^{T} \\
& W=[W(0), W(1), \ldots \ldots \mathrm{W}(\mathrm{N}-1)]^{T} \\
& H=[H(0), H(1), \ldots \ldots \mathrm{H}(\mathrm{N}-1)]^{T}=\operatorname{IFFT}(h) \\
& F=\left[\begin{array}{ccc}
W_{N}^{00} & \ldots & W_{N}^{0(N-1)} \\
\vdots & \ddots & \vdots \\
W_{N}^{(N-1) 0} & \ldots & W_{N}^{(N-1)(N-1)}
\end{array}\right]
\end{aligned}
$$




$$
W_{N}^{n k}=\frac{1}{N} e^{-j 2 \pi\left(\frac{n}{N}\right) k}
$$

If the channel vector $h$ and noise vector $\mathrm{W}$, are uncorrelated then the channel estimate $h$ using MMSE approach in the frequency domain [11] is given by

$$
H_{M M S E}=F R_{h Y} R_{Y Y}^{-1} Y
$$

Where $R_{h Y}=E\{h Y\}=R_{h h} F^{H} X^{H}$

is the Cross correlation matrix between $\mathrm{h}$ and $\mathrm{Y}$

$R_{Y Y}=E\{Y Y\}=X F R_{h h} F^{H} X^{H}+\sigma^{2} I_{N}$

is the auto correlation matrix , and $\sigma^{2}$ represents the noise Channel estimate $h$ using LS approach in the frequency domain is given by

$$
H_{L S}=X^{-1} Y
$$

Which minimizes

$$
(Y=X F h)^{H}(Y=X F h)
$$

In pilot channel estimation, the data can be estimated at data-subcarriers using channel information at pilot subcarriers. This can be done with efficient interpolation techniques. [7]

\section{A. LINEAR INTERPOLATION}

In this method two successive pilot subcarriers are used to find the channel response for data carriers. The channel estimation of data carriers is given by

$$
\begin{aligned}
& H_{\theta}(k)=H_{\theta}(m D+l) \quad 0 \leq l<D \\
& =\left(H_{p}(m+1)-H_{p}(m) \frac{l}{D}+H_{p}(m)\right.
\end{aligned}
$$

where $\mathrm{D}$ is the spacing between two adjacent pilot subcarriers and $\mathrm{H}_{\mathrm{p}}(\mathrm{m})$ is the value of the received pilot subcarrier at frequency $\mathrm{m}$. The frequency response of the channel is considered as a straight line between two adjacent pilot carriers.

\section{B. CUBIC INTERPOLATION}

The third degree polynomial can be obtained in the cubic interpolation using four known points. The values between reference values can be obtained with the following expression

$$
P_{1}(k)=a_{3} K^{3}+a_{3} K^{3}+a_{1} K+a_{0}
$$

where $a_{k}$ are the coefficients of the polynomial and $k$ is the frequency index.

When the range of interpolation is larger than the range enclosed by first four reference points, then it is essential to obtain a second polynomial, using the subsequent four points. It is significant to assure that the first and second order derivative must be considered in the computation of the coefficient in the interpolation.

\section{CUBIC-SPLINE INTERPOLATION}

The transfer function of each subcarrier is approximated to the third order polynomials with respect to $1 / \mathrm{L}$. Estimation is done by obtaining coefficients of the polynomials by means of four adjacent reference signals and their second order derivatives. Hence, the estimate of a subcarrier is

$\mathrm{H}^{\wedge}(\mathrm{k})=\mathrm{A}\left(\frac{1}{\mathrm{~L}}\right) \mathrm{H}_{\mathrm{r}}(\mathrm{m})+\mathrm{B}\left(\frac{1}{\mathrm{~L}}\right) \mathrm{H}_{\mathrm{r}}(\mathrm{m}+1)+\mathrm{C}\left(\frac{1}{\mathrm{~L}}\right) \mathrm{z}(\mathrm{m})+\mathrm{D}\left(\frac{1}{\mathrm{~L}}\right) \mathrm{z}$

Where $A\left(\frac{1}{L}\right), B\left(\frac{1}{L}\right), C\left(\frac{1}{L}\right)$ and $D\left(\frac{1}{L}\right)$

are constants determined by $\left(\frac{1}{\mathrm{~L}}\right) \mathrm{z}(\mathrm{m})=\mathrm{H}_{\mathrm{r}}(\mathrm{m})$

$\mathrm{H}_{\mathrm{r}}(\mathrm{m})$ is the second order derivative of the transfer function of the $\mathrm{m}^{\text {th }}$ reference signals, then all the second order derivatives can be given by

$$
\mathrm{Z}=\mathrm{K}^{-1} \mathrm{~V}
$$

Where

$Z=[z(m-(K / 2)+1), ., z(m 0,, z(m-K / 2)]$

$\mathrm{X}$ and $\mathrm{V}$ are numerator and denominator matrix of the second order derivatives, respectively. Therefore, additional computations to find the second order derivatives of the transfer function are required

\section{DFT BASED CHANNEL ESTIMATION}

The DFT-based channel estimation [12] technique has been derived to increase the performance of LS or MMSE channel estimation by removing the effect of noise outside the maximum channel delay. Let $\mathrm{H}[\mathrm{k}]$ denotes the estimate of channel gain at the kth subcarrier, obtain by either LS or MMSE channel estimation method. Taking the IDFT of the channel estimate

$\operatorname{IDFT}\{\widehat{\mathrm{H}}[\mathrm{k}]\}=\mathrm{h}(\mathrm{n})+\mathrm{z}(\mathrm{n}) \sim \hat{\mathrm{h}}(\mathrm{n}), \mathrm{n}=0,1 \ldots \mathrm{N}-1$

Where $\mathrm{z}(\mathrm{n})$ denotes the noise component in the time domain. Ignore the coefficient $\mathrm{h}^{\wedge}(\mathrm{n})$ that contain the noise only, then the coefficients for the maximum channel delay $\mathrm{L}$ is given as

$$
\begin{gathered}
\hat{\mathrm{h}}_{\mathrm{DFT}}(\mathrm{n})=\mathrm{h}(\mathrm{n})+\mathrm{z}(\mathrm{n}), \mathrm{n}=0,1 \ldots \mathrm{L}-1 \\
=0, \text { Otherwise }
\end{gathered}
$$

and transform the remaining $\mathrm{L}$ elements back to the frequency domain as follows

$\widehat{\mathrm{H}}_{\mathrm{DFT}}(\mathrm{K})=\operatorname{DFT}\left\{\hat{\mathrm{h}}_{\mathrm{DFT}}(\mathrm{n})\right\}$

\section{RESULTS}

In the OFDM system, the transmitted bits have been modulated by different modulation techniques like BPSK or QPSK.

TABLE I.

SIMULATION PARAMETERS

\begin{tabular}{|l|l|}
\hline Parameters & Specifications \\
\hline FFT length & 1024 \\
\hline Number of subcarriers(active) & 64 \\
\hline Number of data carriers & 48 \\
\hline Number of pilot carriers & 4 \\
\hline Sampling frequency & $20 \mathrm{MHZ}$ \\
\hline Number of carriers for cyclic prefix & 16 \\
\hline Modulation & BPSK,QPSK \\
\hline Channel Model & Rayleigh fading \\
\hline
\end{tabular}


Modulated data will be converted into parallel form where each bit is being multiplied with the carrier in the IFFT block, so that the output of the IFFT block (i.e. Parallel data) is converted into serial form. Then by adding cyclic prefix the required signal can be obtained. Simulation parameters considered to implement OFDM system are shown in Table I.
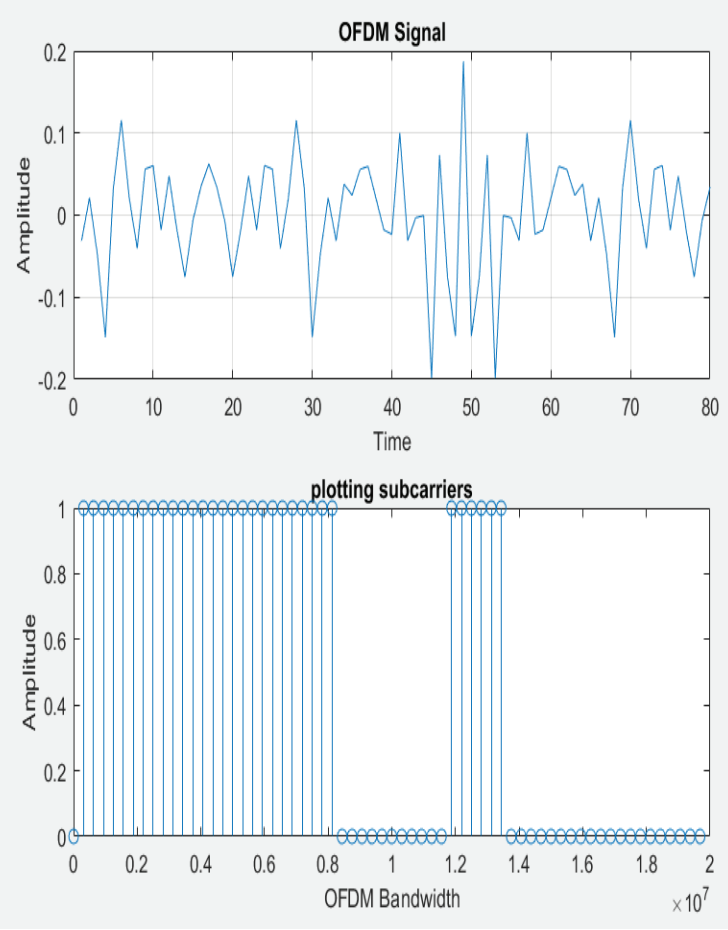

Figure 2. Generation of OFDM signal with 64 subcarriers

According to the WLAN standards, 64 subcarriers are considered as data carriers, according to the 4 pilot subcarriers are transmitted. Each symbol on subcarrier indices can be represented as shown in the figure 4.1 where $\mathrm{Y}$ axis represents the no of subcarriers i,e., from 0 to 63 and $\mathrm{X}$ axis represents the time taken by each subcarrier to be on the frame (frame time ). The bandwidth of the OFDM signal is $20 \mathrm{MHz}$ which is divided by each subcarrier $(0.3125 \mathrm{MHz})$. Each symbol duration will be $3.2 \mu$ s.

Various Pilot Channel Estimation techniques are implemented for OFDM system and channel estimates are evaluated using interpolation techniques. The performance metrics such as MSE, BER and Computation time are calculated and discussed in sections A, B and C.

\section{A. Performance Analysis of Different Channel Estimation Techniques using Linear / Spline Interpolation:}

The channel estimates obtained by using the various channel estimation methods with and without DFT technique are compared and plotted at various subcarrier indices are shown in the Figure.3..and Table.2.
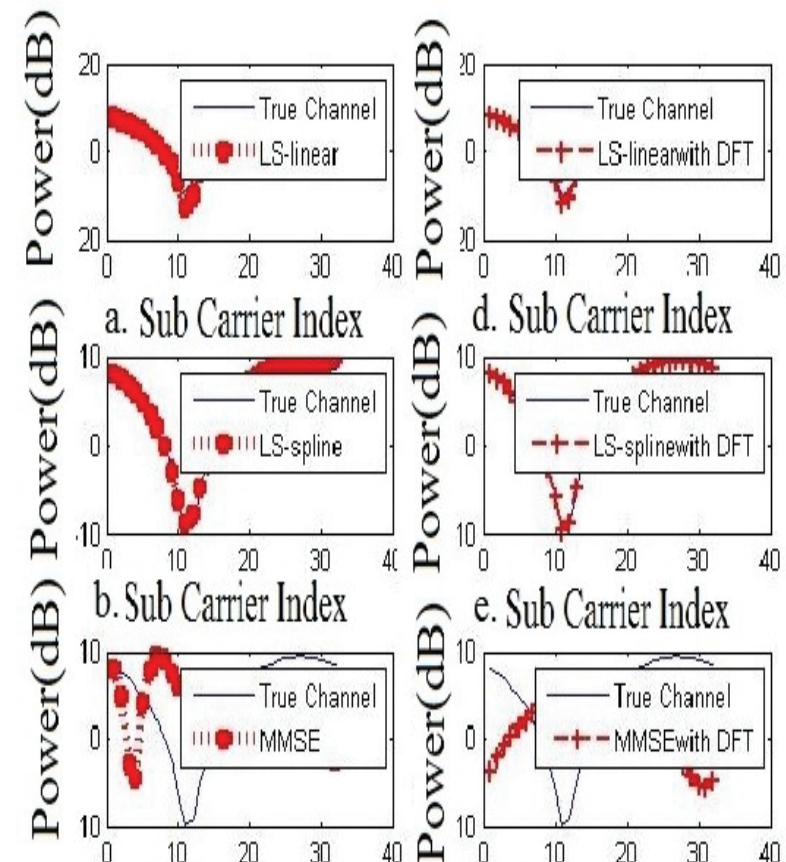

c. Sub Carrier Index

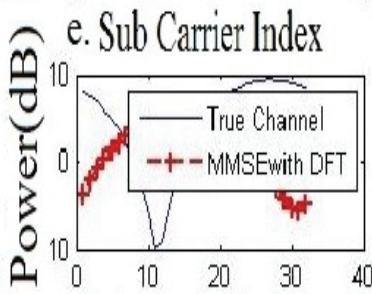

f. Sub Carmer Index

Figure 3. Performance analysis of different channel estimation techniques using Linear/Spline interpolation

TABLE II.

POWER (DB) AT VARIOUS SUBCARRIER INDICES WITH AND WITHOUT DFT

\begin{tabular}{|c|c|c|c|c|c|}
\hline $\begin{array}{l}\text { Sub } \\
\text { carrier } \\
\text { index }\end{array}$ & $\begin{array}{l}\text { True } \\
\text { channel }\end{array}$ & $\begin{array}{l}\text { LS Linear } \\
\text { (without } \\
\text { DFT) }\end{array}$ & $\begin{array}{l}\text { LS } \\
\text { Spline } \\
\text { (with } \\
\text { DFT) }\end{array}$ & $\begin{array}{l}\text { MMSE } \\
\text { Linear } \\
\text { (without } \\
\text { DFT) }\end{array}$ & $\begin{array}{l}\text { MMSE } \\
\text { Linear } \\
\text { (with } \\
\text { DFT) }\end{array}$ \\
\hline 5 & 4 & 4 & 4 & 2.5 & -2 \\
\hline 15 & 4.5 & 4.5 & 4.5 & 5.5 & 4 \\
\hline 25 & -10 & -11 & -10 & 1 & -1 \\
\hline 27 & -15 & -13 & -15 & 1 & 1 \\
\hline 30 & -5 & -5 & -4 & 1 & -5 \\
\hline
\end{tabular}

The channel response is computed for LS-Linear, LSSpline and MMSE approach then compared with true channel response and plotted in in Figure.3.a, b, c. Figure3.a. depicts that LS-linear channel response is close to True channel response. DFT is combined with LS-Linear, LS-Spline, MMSE techniques and channel response is plotted in Figure 3.d, e, f. The Figure 3. and Table. II. Shows that the DFT-based channel estimation method improves the performance of channel estimation since it is close to the true channel response.

\section{B. MSE VS EBNo CHARACTERISTICS OF PCE TECHNIQUES}

MSE is calculated for LS, MMSE \&LMMSE (Linear MMSE) approaches and MMSE technique is combined with TD (Time Duplexing) \&TDD (Time Division Duplexing) 
techniques. MSEs of various techniques are compared and plotted MSE vs EbNo characteristics shown in Figure.4.

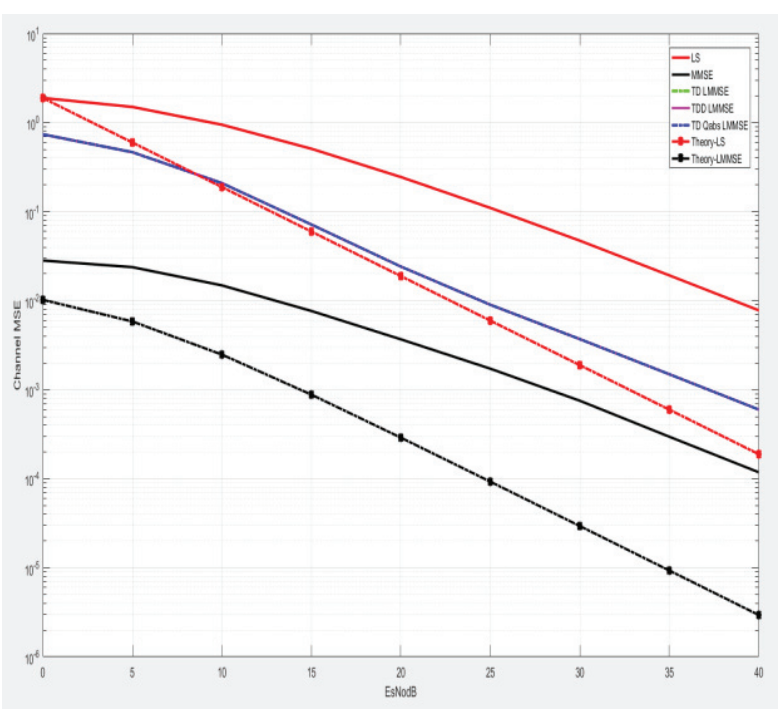

Figure 4. MSE vs EbNo Characteristics for different Pilot Channel estimation techniques

The MSE vs EbNo characteristics shows that LMMSE with TD approach gives minimum MSE. depends on the channel estimate equations, transfer function, impulse response, cross correlation, and channel energy.

The time required to compute Mean Square Error (MSE) is calculated for various Pilot Channel Estimation techniques and shown in Table III. are computed based on the time required for matrix multiplications, matrix inversions, DFT Computation.

\section{Conclusions}

PCE Estimation method is implemented for OFDM system. At the transmitter, equispace and equipower pilots are inserted in the data. The pilots have been designed and inserterd.in the data. The modulated signal and pilots are transmitted through Rayleigh fading Channel. At the receiver these pilots are extracted, and extracted pilot $\mathrm{s}$ are used to estimate the channel using various techniques like Linear, cubic interpolations.

TABLE III.

COMPARISION O COMPUTATION TIME(NS)

\begin{tabular}{|c|l|l|l|l|l|l|l|}
\hline $\begin{array}{l}\text { SNR } \\
(\mathrm{db})\end{array}$ & LS & Theory LS & MMSE & Theory MMSE & TD MMSE & TDD MMSE & TDQabs \\
\hline 0 & $192 \times 10^{-2}$ & $195 \times 10^{-2}$ & $433 \times 10^{-2}$ & $404 \times 10^{-2}$ & $430 \times 10^{-2}$ & $476 \times 10^{-2}$ & $453 \times 10^{-2}$ \\
\hline 5 & $168 \times 10^{-2}$ & $177 \times 10^{-2}$ & $413 \times 10^{-2}$ & $399 \times 10^{-2}$ & $380 \times 10^{-2}$ & $465 \times 10^{-2}$ & $425 \times 10^{-2}$ \\
\hline 20 & $170 \times 10^{-2}$ & $172 \times 10^{-2}$ & $394 \times 10^{-2}$ & $386 \times 10^{-2}$ & $419 \times 10^{-2}$ & $438 \times 10^{-2}$ & $417 \times 10^{-2}$ \\
\hline 30 & $169 \times 10^{-2}$ & $173 \times 10^{-2}$ & $351 \times 10^{-2}$ & $403 \times 10^{-2}$ & $400 \times 10^{-2}$ & $434 \times 10^{-2}$ & $424 \times 10^{-2}$ \\
\hline 40 & $171 \times 10^{-2}$ & $171 \times 10^{-2}$ & $371 \times 10^{-2}$ & $297 \times 10^{-2}$ & $416 \times 10^{-2}$ & $460 \times 10^{-2}$ & $422 \times 10^{-2}$ \\
\hline
\end{tabular}

For a given SNR, MMSE estimation performs better because of the mean square error for particular values of SNR [Decibels] decreases quickly in the required elapsed time when compared to the other estimation techniques. The complexity of MMSE estimator is large than LS estimator but gives better performance in comparison to LS estimation.

\section{COMPUTATIONAL TIME}

The pilot channel estimation technique performance can also be expressed in terms of computational complexity. In this section all estimators' computational complexities are evaluated. The computational complexity for LS, MMSE algorithm
The resulting MSE for MMSE with TD approach is reduced by $3.16 \%$ at $0 \mathrm{db}$ SNR which is better with the existing techniques. Finally, the time required to compute MSE is also evaluated for LS and MMSE approaches and MMSE technique computationally complex with least MSE for OFDM system.

\section{REFERENCES}

[1] L. J. Cimini, Jr., B. Daneshrad, and N. R. Sollenberger, "Clustered OFDM with transmitter diversity and coding," in Proc. 1996 IEEE Global Telecommunications Conf., London, U.K., pp. 703-707.

[2] L. J. Cimini, Jr., "Analysis and simulation of a digital mobile channel using orthogonal frequency division multiplexing," IEEE Trans. Commun., vol. COM-33, pp. 665-675, July 1985 
[3] Theodore S. Rappaport, "Wireless Communications: Principles and Practice", 2 nd Edition,

[4] Prentice Hall, 2002. A. R. S. Bahai and B. R. Saltzberg, Multi-Carrier Digital Communications: Theory and Applications of OFDM: Kluwer Academic/Plenum, 1999.

[5] Sinem Coleri, Mustafa Ergen, Anuj Puri, and Ahmad Bahai,' Channel Estimation Techniques Based on Pilot Arrangement in OFDM Systems'IEEE Transactions On Broadcasting, Vol. 48 , No. 3, September 2002..

[6] Chengyang Li, Sumit Roy, 'Subspace-Based Blind Channel Estimation for OFDM by Exploiting Virtual Carriers'IEEE Transactions On Wireless Communications, Vol. 2, No. 1, January 2003.

[7] J.-J. van de Beek, O. Edfors, M. Sandell, S. K. Wilson, and P. O. Borjesson, "On channel estimation in OFDM systems," in Proc. IEEE 45th Vehicular Technology Conf., Chicago, IL, Jul. 1995, pp. 815-819.

[8] M. Hsieh and C.Wei, "Channel estimation for OFDM systems based on comb-type pilot arrangement in frequency selective fading channels," IEEE Trans. Consumer Electron., vol. 44, no. 1, Feb. 1998 .
[9] O. Edfors, M. Sandell, J.-J. van de Beek, S. K. Wilson, and P. O. Brjesson, "OFDM channel estimation by singular value decomposition," IEEE Trans. Commun., vol. 46, no. 7, pp. 931-939, Jul. 1998.

[10] Meng-Han Hsieh , Che-Ho Wei "Channel Estimation For OFDM Systems Based On Comb-Type Pilot Arrangement In Frequency Selective Fading Channel" IEEE Transactions On Consumer Electronics, Vol. 44, No. 1, February 1998

[11] Y. Zhao and A. Huang, "A novel channel estimation method for OFDM Mobile Communications Systems based on pilot signals and transform domain processing," in Proc. IEEE 47th Vehicular Technology Conf., Phoenix, USA, May 1997, pp. 2089-2093.

[12] A. V. Oppenheim and R. W. Schafer, Discrete-Time Signal Processing, New Jersey: Prentice-Hall Inc., 1999.

[13] Tang, R.G.; Zhou, X.; Wang, C.Y. A Haar wavelet decision feedback channel estimation method in OFDM systems. Appl. Sci. 2018, 8, 877. [CrossRef] 